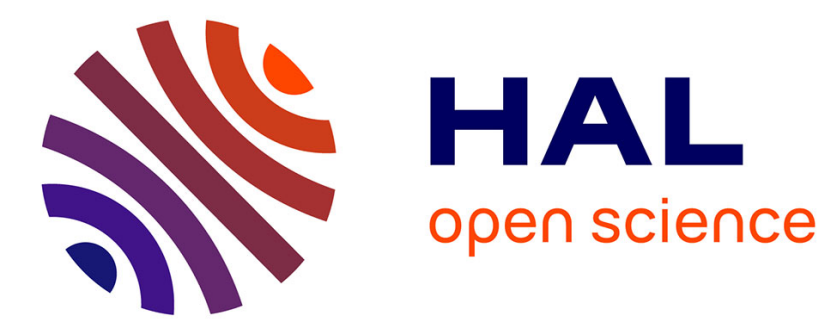

\title{
Sur l'étymologie du nom de l'Averne
}

Romain Garnier

\section{To cite this version:}

Romain Garnier. Sur l'étymologie du nom de l'Averne. Revue de philologie, 2010, 82/1, pp.99-111. hal-00940840

\section{HAL Id: hal-00940840 \\ https://hal.science/hal-00940840}

Submitted on 3 Feb 2014

HAL is a multi-disciplinary open access archive for the deposit and dissemination of scientific research documents, whether they are published or not. The documents may come from teaching and research institutions in France or abroad, or from public or private research centers.
L'archive ouverte pluridisciplinaire HAL, est destinée au dépôt et à la diffusion de documents scientifiques de niveau recherche, publiés ou non, émanant des établissements d'enseignement et de recherche français ou étrangers, des laboratoires publics ou privés. 


\section{SUR L'ÉTYMOLOGIE DU NOM DE L'AVERNE \\ (facilis descensus Auerno)}

\section{Introduction :}

Le nom de l'Averne (lat. Auernum n.), la fameuse entrée des Enfers décrite dans l'Énéide, est totalement immotivé en latin-même. Pour les anciens, ce mot s'explique par un

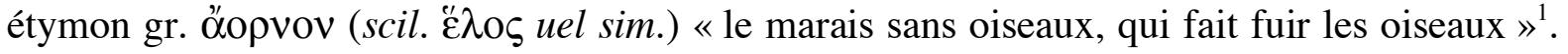

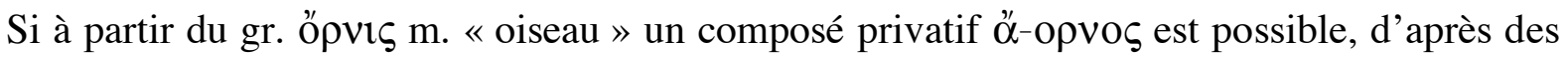

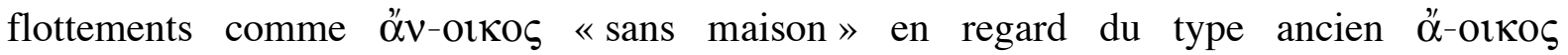
$\left(<* \alpha^{\prime \prime}-F \mathrm{Ol \kappa o \varsigma}\right)^{2}$, il est sans doute plus commode d'expliquer le lat. Auernum par la substantivisation d'un ancien adjectif indiquant une position contrastive dans l'espace, soit le type de lat. infernum n. «enfer» (et inferna n. pl. «les enfers ») en regard de l'adjectif infernus, - $a$, -um. Un fait mérite d'être signalé, c'est que le terme Auernum ne se démarque pas de son dérivé d'appartenance : on dit ainsi loca Auerna et non *loca Auernia ou *loca Auernica. Cela s'explique fort bien si l'on accepte de voir dans le lat. Auernum le neutre d'un adjectif en -ernus (<*-er-ino-) du type de lat. infernus, externus ou supernus. Dans l'étude qui suit, on se propose de remotiver cet adjectif *auernus (<*au-er-ino-) qui doit être un synonyme dialectal de infernus, apparaissant sous une forme fossile dans un toponyme. De fait, il existe un correspondant védique à l'étymon it. com. *aun-ero- «inférieur ». Il s'agit du véd. ávara- "situé en bas, inférieur ». Le nom de l'Averne permettrait ainsi de rétablir une correspondance lexicale ancienne entre l'italique et l'indo-iranien.

\section{1. l'entrée des enfers :}

L'entrée de l'Averne est amplement décrite par Virgile au chant 6 de l'Enéide, aux vers 237 à 241 ,

\section{Spēlunca alta fuit uastōque immānis hiātū}

scrūpea, tūta lacū nigrō nemorumque tenebrīs, quam super haud ulla poterant impūne uolantēs

tendere iter penn̄̄s : tālis sēs( $\bar{e})$ hālitus atrīs

faucibus effundens supera ad conuexa ferēbat.

«Il y avait une caverne profonde, monstrueuse, ouverte en un bâillement énorme, hérissée de rocs, défendue par un lac noir et les ténèbres des bois. Nul oiseau ne pouvait dans son vol passer impunément au-dessus; tel était le souffle qui se dégageait de ces gorges sombres et montait jusqu'aux voûtes célestes. » ${ }^{3}$

\footnotetext{
${ }^{1}$ C'est à dire le marais dont les exhalaisons méphitiques tuent les oiseaux en plein vol.

${ }^{2}$ Ces vues m'ont été suggérées par M. CASEVITZ, à qui je tiens à exprimer ici ma gratitude pour sa riche relecture.

${ }^{3}$ Trad. J. PERRET, CUF, 1978.
} 
Le vers 242, aux prétentions étiologiques (unde locum Grāı̄ dīxērunt nōmine öo « de là vient que les Grecs nommèrent ce lieu ơo $\rho v o \varsigma ~ »{ }^{4}$ ), est généralement tenu pour une maladroite interpolation. Nonius $(14,4)$ reprend à son compte cette étymologie: Auernus...quia odor auibus infestissimus «Le lieu (est nommé) 'Auerne' du fait que son odeur est fatale aux oiseaux ».

De même, Pline, dans son Hist. Nat. (4. 1, 2), évoque « un lieu őopvos et une

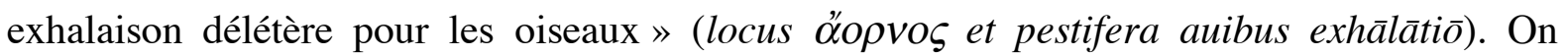
surprend un jeu de mot sous-jacent chez tous ces auteurs qui associent auis « oiseau » à Auernum. C'est là une sorte d'acclimatation latine de la motivation savante du lat. Auernum que les Latins faisaient remonter à un composé privatif $\alpha$-opvo $\varsigma$ «sans oiseaux, où les oiseaux ne vont pas ». Pline évoque de semblables lieux, hors de tout contexte magicoreligieux, dans son Hist. Nat. (2. 93, 207), spīritūs lētālēs...aliubi uolucribus tantum...aliubi prater hominem cēterīs animālibus, nonnunquam et homin̄̄ «(il y a) des exhalaisons mortelles $\langle\ldots\rangle$ tantôt funestes seulement pour les oiseaux $\langle\ldots\rangle$ tantôt pour tous les animaux excepté l'homme, et certaines fois pour l'homme ».

\section{2. étude du lat. Auernum :}

D'un point de vue phonétique, il est tout à fait possible d'admettre pour Auernum un étymon gr. *őopvov. L'émergence d'un - $u$ - de transition pour monnayer une séquence - $\alpha \mathrm{O}^{-}$ est attestée pour le type $\dot{\alpha} o \rho \tau \eta ́$ f. « sac ou besace » qui aboutit à lat. auerta ${ }^{5}$ (<*auorta). Par contre, ce qui est anomal, c'est que l'adjectif d'appartenance du lat. Auernum soit simplement Auernus, - $a$, -um. De fait, on attendrait plutôt quelque chose comme *Auernicus ou bien *Auernius, même pour un mot d'emprunt. Il n'est que de citer Virg., En. 6, 118, nec tē \#

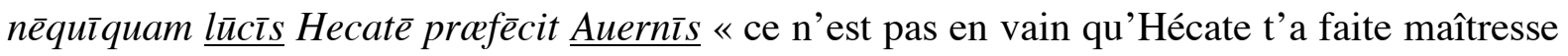
du bois de l'Averne ». De même, on trouve Auerna loca «des lieux semblables à l'Averne » chez Lucr. $(6,738)$.

3. nouvelle orientation étymologique :

Il est possible de trouver une tout autre manière de rendre compte de ces faits. Le lat. Auernum est peut-être un terme indigène, secondairement élucidé par un étymon grec de circonstance. Si c'est un ancien adjectif substantivé du type hībernum, on ne s'étonnera plus qu'il ne possède pas de dérivé d'appartenance. La forme doit s'analyser comme un ancien adjectif spatial contrastif en -ernus (soit le type supernus $\sim$ infernus). En regard du neutre

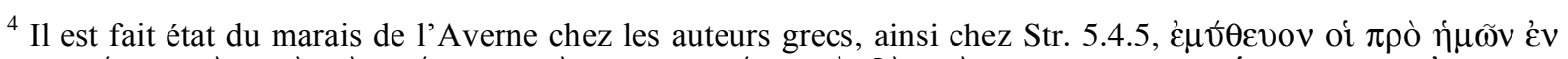

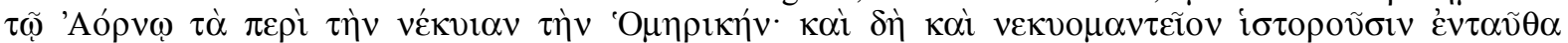

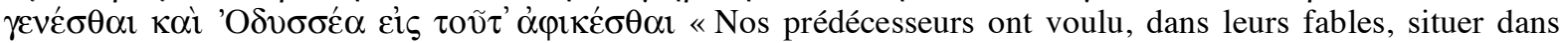
l'Averne l'épisode de la vékvi $\alpha$ homérique : ils rapportent qu'on y pratiquait jadis la nécromancie et que c'est là que se rendit Ulysse ».

${ }^{5}$ Forme attestée dans le Codex Theodosianus 8, 5, 47.
} 
pluriel inferna, -ōrum « les enfers ${ }^{6}$ (Tac. H. 5, 5) on emploie infernus, - $a$, -um « des enfers, infernal » en fonction d'adjectif d'appartenance, ainsi chez Virg., En. 6, 106, infern̄̄ iānua rēgis \# «la porte du roi des enfers ». Le dérivé Auernālis « de l'Averne » ${ }^{7}$ serait donc un dérivé de dérivé, à l'instar du type infernālis « infernal » fait sur le neutre substantivé inferna « les enfers » ou de hībernālis « hivernal » fait sur hībernum n. « la période hivernale ».

Le terme Auernum ou Auerna (loca) doit être en propre une ancienne désignation des enfers, en tant que lieux inférieurs. Chez Virgile, il est loisible de relever le contraste entre En. 6, 126, facilis descensus Auernō \# « il est facile de descendre en l'Averne » et En. 6, 128 sed reuocāre gradum, superāsque ēuādere ad aurās \# hōc opus, hic labor est « mais revenir sur ses pas, se retrouver libre sous les souffles d'en haut, voilà ce qui est l'affaire et qui demande effort » (Trad. J. PERRET, CUF, 1978). On peut supposer une opposition entre un adjectif *auernus «inférieur» (substantivé au sens d'enfer) et son antonyme superus (supernus) « qui se trouve en haut ». On partirait d'un étymon it. com. *aun-er-ino- apparenté au véd. ávara- « inférieur». Les loca auerna ${ }^{8}$ seraient tout simplement des loca inferna.

4. dans quel système s'inscrirait un étymon it. com. *aú-er-ino- « situé en bas »?

Il existe en latin tout un système d'adjectifs indiquant une position dans l'espace. Ils sont bâtis sur un adverbe, et offrent l'aspect de comparatifs ou de superlatifs, indiquant respectivement une position contrastive ou absolue dans l'espace ${ }^{9}$ :

\begin{tabular}{|c|c|c|}
\hline adverbe spatial & dérivé « comparatif » & dérivé « superlatif » \\
\hline $\begin{array}{c}\text { inter }(<* \text { enter }) \\
\text { « entre, à l'intérieur » }\end{array}$ & $\begin{array}{c}\text { *interus }(<* \text { ent-er-ó- }) \\
\text { internus }(<* \text { enter-inó- }) \\
\text { (interior })\end{array}$ & $\begin{array}{c}\text { intimus }(<* \text { ent-m-ó- }) \\
( \pm \text { véd. antamá- })\end{array}$ \\
\hline $\begin{array}{l}e x\left(<* e \widehat{g}^{h}-s\right) \\
\ll \text { hors de } »\end{array}$ & $\begin{array}{c}\text { *exterus }\left(<* \text { e } \widehat{g}^{h}-s \text {-ter-ó- }\right) \\
\text { externus }\left(<* e \widehat{g}^{h}-s \text {-ter-inó- }\right) \\
(\text { exterior })\end{array}$ & extimus $\left(<* e g^{h}-s-t m-o ́-\right)$ \\
\hline $\begin{array}{c}* i n f e r\left(<* n d^{h} \text {-ér }\right) \\
\text { « en bas » }\end{array}$ & $\begin{array}{c}\text { inferus }\left(<^{*} n d^{h} \text {-er-ó-) }\right. \\
\text { infernus }\left(<* n d^{h} \text {-er-i-nó-) }\right. \\
\text { (inferior) (véd. ádhara-) }\end{array}$ & $\begin{array}{l}\text { infimus }\left(<^{*} n d^{h}-m-o ́-\right) \\
\text { (véd. adhamá-) }\end{array}$ \\
\hline $\begin{array}{c}\text { super }(<* \text { upér-i }) \\
\text { « en haut } »\end{array}$ & $\begin{array}{c}\text { superus }(<* \text { uper-ó- }) \\
\text { supernus }(<* \text { uper-i-nó- }) \\
(\text { superior })\end{array}$ & $\begin{array}{c}\text { summus }(<*(\text { s)up-m-ó- }) \\
( \pm \text { véd. upamá- })\end{array}$ \\
\hline
\end{tabular}

\footnotetext{
${ }^{6}$ Noter en ce sens le doublet Auerna, -ōrum n. pl. attesté chez Virg., En. 3, 442, Auerna sonantia siluīs \# «l'Averne qui résonne du bruit de ses forêts ».

${ }^{7}$ Horace, Ep. 5, 26 et Ovide, M. 5, 340.

${ }^{8}$ Qu'il conviendrait sans doute d'écrire sans majuscule chez Lucrèce $(6,738)$.

${ }^{9}$ Pour un exposé des faits exhaustif, consulter Leumann (1977:317) et WACKERNAGEL (AiGr. II, $\left.2: 217\right)$.
} 


\begin{tabular}{|c|c|c|}
\hline $\begin{array}{c}* a u(<* a u)^{10} \\
\text { « de haut en bas, au loin » }\end{array}$ & $\begin{array}{l}\text { *auernus (<*auner-i-nó-) } \\
\text { « situé en bas » ( } \pm \text { véd. ávara-) } \\
\text { (av. aorā adv. « vers le bas ») }\end{array}$ & $\varnothing$ \\
\hline
\end{tabular}

5. le préverbe latin $a u-:$ syntaxe et phraséologie

Tout l'enjeu est de savoir s'il est permis de mettre en relation le préverbe $a u$ - du latin (lequel est fort rare) avec l'adjectif contrastif *auerno- « situé en bas, inférieur ». On sait que le véd. áva ${ }^{11}$ signifie « de haut en bas » et « au loin ». Sémantiquement, il est loisible de rapprocher le lat. $d \bar{e}-$ « de bas en haut» qui sert aussi à marquer l'éloignement, ainsi dans le type dé-fugiō «s'enfuir »(fr. dé-camper, dé-guerpir).

\section{1. lat. auferō :}

Il ne saurait être question de dénier toute existence au préverbe latin $a u$ - en y voyant l'allomorphe spirantisé de $a b-{ }^{12}$. On ne saurait admettre qu'une forme *af-ferō (<*ab-fer-) eût été non-conforme à la phonétique latine, à en juger par offerō (<*ob-fer-). On ne saurait non plus se fonder sur Cicéron ${ }^{13}$ pour rejeter la possibilité d'une variante récente $* a b$-fer- ${ }^{14}$ qui se distinguât nettement de l'homonymique affer- (<*ad-fer-) en réintroduisant le préverbe $a b$ - de façon analogique.

Du reste, le traitement phonétique standard eût donné lat. **āferō comme on a $\bar{a} f u \bar{u}$, $\bar{a}$ forem et âfutūrus en regard de ab-sum et ab-esse. On ne peut se contenter de conclure que «The question of why this analogy did not reach auferō and aufugiō must remain unanswered ${ }^{15}$. Il est manifeste que le préverbe archaïque du lat. au-ferō «emporter vivement ${ }^{16}$ représente un isolat morphologique à l'intérieur du lexique latin, mais ce préverbe rare et résiduel possède des correspondants dans d'autres langues, ainsi le r. u-berët

\footnotetext{
${ }^{10}$ Attesté en latin seulement sous forme du préverbe $a u$ - marquant la séparation (ainsi dans au-ferō « emporter » qui correspond au r. u-berët « il emportera »). Le hitt. $\bar{u}$ - $(<$ anatolien com. *au) interdit de poser une laryngale initiale de timbre $* h_{2^{-}}$(MELCHERT $\left.1984: 61\right)$, ce dont de VAAN (2003-04:2) semble ne pas s'être avisé en posant un étymon $* h_{2}$ eun. Noter que le véd. áva (gouvernant l'ablatif) signifie « en bas, vers le bas » et « au loin » (noter $R V$ 1. 129, 6 ava-tarám (adv.) «plus loin »). La notion de descente enferme la valeur de déchéance et de perte, qui explique l'emploi de *aú comme préfixe privatif qu'on relève dans le v.-sl. u-bogŭ «pauvre » (en propre « sans part, privé de part ») qui s'oppose ainsi au type bog-atŭ « pourvu d'une bonne part, riche ».

${ }^{11}$ Le second $-a$ du véd. $a ́ v-a$ doit s'expliquer par úpa à qui il emprunterait sa finale et ne saurait remonter audelà de l'indo-iranien (l'avestique présente ainsi auua.baraiti "emporter » en regard du véd. áva-bharati). DüNKEL (1988) pose un étymon i.-e. * a uno qu'il distingue du groupe de la particule $* h_{2} u$ « en revanche, mais ».

12 Contra : de VAAN (2008: 19-20). Ce dernier offre en revanche un bon schéma explicatif pour la sonorisation de lat. $a b$. On peut partir d'it. com. *apo-dounk-e/o- pour aboutir à lat. abdūcō (de VAAN, 2003$04: 4$, n. 4). Cette explication figure déjà chez SOMMER (1914:275).

${ }^{13}$ Cic., Or. 158, Quid, quod etiam abfugit turpe uīsum est et abfer nōluērunt, aufugit et aufer māluērunt? «Qui plus est, on a trouvé affreux abfugit et on a refusé abfer, préférant aufugit et aufer ».

${ }^{14}$ Du type ab-fuat « absit » (Front., Amic., 1. 14, 2) ou bien ab-forem pour âforem.

15 de VAAN (2003-04:6).

${ }^{16}$ Noter Pl., Aul., 638 aufer cauillam «porte au loin (=cesse) tes plaisanteries » et P1., As., 469 aufer tē hinc « ôte toi d'ici! ». Virgile emploie le tour fugāx aufertur $(E n .11,713)$ « il s'échappe à vive allure ».
} 
« il emportera $»^{17}$ ou bien le véd. áva-BHR- « enlever, ôter » (la tête du corps ${ }^{18}$ ) qu'on relève en $R V$ 10. 171, 2ab,

\section{t(u)vám makhásya dódhatạ̣ śíro (á)va tvacó bharah}

«c'est toi qui as séparé de (son) corps ${ }^{19}$ la tête de Makha qui tremblait ${ }^{20}$ »

\section{2. lat. autumnum n. « automne »:}

Quelle qu'en soit l'origine, le préverbe i.-e. *aun marque une séparation violente, incluant la notion d'arrachage. Il a fourni à l'italique une désignation de la moisson. Récemment (RIX $1997^{21}$ ), il vient d'être démontré que le lat. autumnum n. longtemps réputé d'origine étrusque repose sur un adjectif en *-ino-indiquant le temps : on poserait un ancien syntagme *autum(i)num (tempus) «période de la récolte ${ }^{22}$ » comme on a hībernum (tempus) «période hivernale ${ }^{23}$. Le dérivé *autum-ino- «(temps) de la récolte ${ }^{24}$ postule un ancien

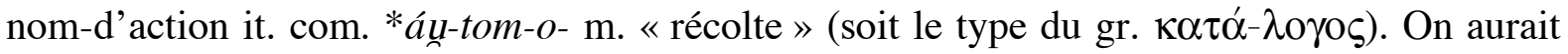
ici le reflet d'un ancien syntagme i.-e. *aú- temh ${ }_{1^{-}}$« arracher, récolter ». La période de la récolte fournit au germanique une désignation de l'automne: l'all. Herbst (v.h.a. harbist)

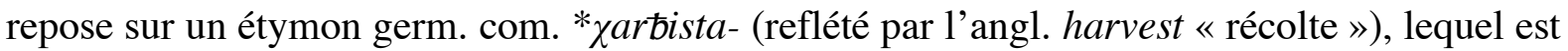

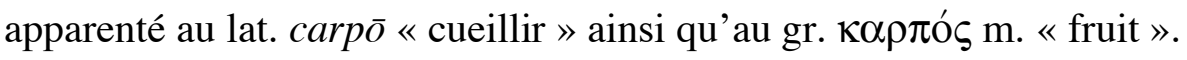

\section{3. lat. aufugiō :}

Le préverbe $a u$ - du lat. au-fugiō «s'échapper vivement » répond étymologiquement au préverbe $u$ - du v.-sl. $u$-běgQ $u$-běžati «s'enfuir ${ }^{25}$ qui reflète la racine $* b^{h} e g^{\underline{u}}$ 《 fuir ${ }^{26}$.

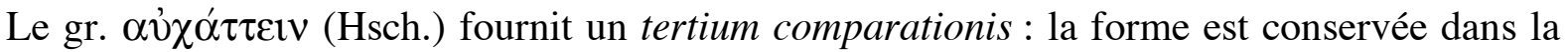

\footnotetext{
${ }^{17}$ Noter le sens du réfléchi $u$-berët-sja «il rangera»(ses affaires). L’imperfectif u-birát'-sja « faire son paquet » s'emploie au sens familier de « décamper» (ainsi relève-t-on chez Pouchkine, La fille du capitaine, chap. XIII, ubirájte-s'! « ouste, fichez le camp ! »). Non-réfléchi, u-birát' signifie « rentrer la récolte ».

${ }^{18}$ Ce passage rappelle fort l'emploi que fait Virgile du verbe auferō dans l'En., 322-333, tum caput ipsī aufert dominō truncumque relīquit \# sanguine singultantem «puis du maître lui-même il fait tomber la tête, laissant tomber le tronc d'où le sang jaillit par saccades » (il s'agit de Rhamnès, tué dans son sommeil par Nisus).

${ }^{19}$ Le nom-racine tvác- f. «peau » désigne ici le « corps » selon Sāyaṇa (litt. : «enveloppe du corps »). Noter que le hit. tuekka- (<*túék-o-) signifie « corps, personne».

${ }^{20}$ Le participe intensif de la racine $D H \bar{U}$ - «s'agiter »s'emploie en contexte indraïque pour décrire le démon qui se débat. On rapprochera $R V$ 8. 6, 6abc, ví cid vrtrásya dódhato / vájreṇa śatáparvaṇā / śíro bibheda vrșnínā « avec sa virile massue aux cent bosses (Indra) a arraché (ví BHID-) la tête de Vṛtra qui tremblait».

${ }^{21}$ Il est loisible d'en lire le compte-rendu fait par M. EGETMEYER dans la $C E L 3$ (=R. Ph. 79/2, 2005, 331).

${ }^{22}$ On comprend ainsi les graphies fautives de type auctumnum (citées dans le $D E L L: 61$ ) qui reflètent une étymologie synchronique basée sur le verbe augeō « fournir la croissance » (P.-Fest. 21, 27, autumnum quidam dictum existimant quod tunc maxime augeantur hominum opes, coactis agrorum fructibus).

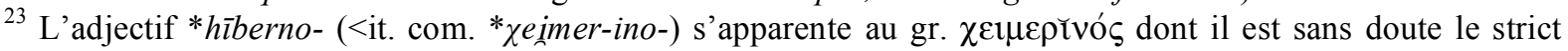
cognat. Le dossier complet figure chez SzMERENYI (1991). Le traitement phonétique, complexe, peut

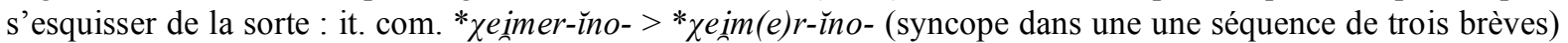
d'où proto-lat. *heifrino- $>$ *heißßrino- $>$ *hībrĭno- > hïbernus « hivernal ».

${ }^{24}$ Soit le type nocturnus « qui se passe pendant la nuit».

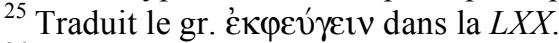

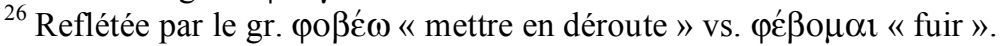


glose $\alpha \hat{\chi} \chi \alpha \dot{\tau} \tau \varepsilon ı v \cdot \dot{\alpha} v \alpha \chi \omega \rho \varepsilon \tilde{v} v$ "s'échapper ${ }^{27}$. En l'espèce, il semble difficile de déterminer ici quelle racine formait jadis syntagme avec l'adverbe i.-e. *au « de haut en bas, au loin» pour donner un verbe «s'enfuir» (cf. lat. dée-fugiō «s'enfuir» en regard du préverbe $d \bar{e}^{28}$ qui veut dire en propre « de haut en bas »). Chaque langue emploie la racine vivante qui veut dire « fuir ». Seule est restituable la communauté d'emploi de ce préverbe archaïque d'après le lat. au-fugiō, le v.-sl. u-b̌̌go et le gr. $\alpha{ }^{-} \chi \alpha \dot{\alpha} \tau \tau \varepsilon \iota v^{29}$.

\section{4. problèmes sémantiques de l'adverbe i.-e. *au « au loin » et « auprès »}

L'adverbe i.-e. *au revêt les sens a priori contradictoires de « au loin » et « auprès ». La préposition russe $u$, quand elle gouverne le génitif, indique nettement la provenance dans un tour comme vzjat' knígu u továrišča « emprunter un livre à un camarade » et la proximité dans le tour sidét' $u$ okná « être assis près de la fenêtre ».

De même, si l'av. аииа-BAR-veut dire emporter au loin dans le Vìdèvdāt 8. 2, аииa aêtam 'ristam ${ }^{30}$ baraiian (avec tmèse) «puissent-ils emporter au loin ${ }^{31}$ ce défunt », auиa.BAR- revêt le sens symétrique d'apporter (gouvernant le génitif ${ }^{32}$ ) en $Y$. 65. 2,

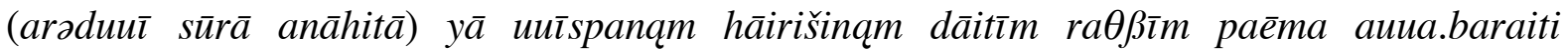
«(Arəduū Sūrā Anāhitā ${ }^{33}$ ) qui apporte à toutes les femmes un flot de lait conforme à la nature (du nourrisson) ${ }^{34}$ et régulier ${ }^{35} »$.

De fait, la réciprocité sémantique de l'adverbe i.-e. *au « au loin » et « auprès » ${ }^{36}$ se laisse sans peine ramener à un unique sens spatial, marquant un mouvement (rapide) du haut vers le bas ${ }^{37}$. Précisé par un ablatif d'origine, un tel mouvement peut aboutir à l'idée

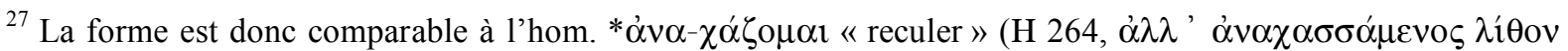

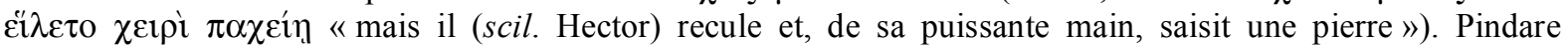

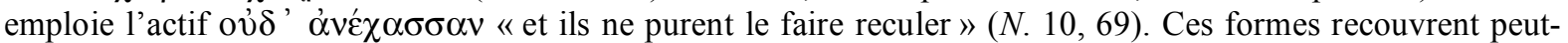
être un couple ancien $* \dot{\alpha} F-\varepsilon-\chi \alpha \dot{\alpha} \sigma \sigma \alpha \tau$ o « il recula » vs. ${ }^{*} \dot{\alpha} F-\varepsilon^{-} \chi \alpha \sigma \sigma \varepsilon$ « il fit reculer » qu'on aurait modernisé au moyen du préverbe $\alpha$ ó $\alpha$ - plus vivant.

${ }^{28}$ Pour l'état complet de la question, consulter l'étude de J.-P. BRACHET (1998).

${ }^{29} \mathrm{La}$ formation du thème de présent $\alpha v_{\chi} \alpha \dot{\tau} \tau \varepsilon \imath$ « fuir » est obscure : c'est peut-être l'allomorphe crétois en

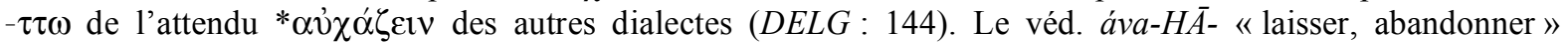
$\left(<* a ́ u-g^{h} e h_{1^{-}}\right)$en représenterait peut-être la contrepartie transitive.

${ }^{30}$ En propre ${ }^{i}$ rista- est le participe du verbe $R A \bar{E} \Theta$ - « aller, passer » qui est un euphémisme pour « mourir ».

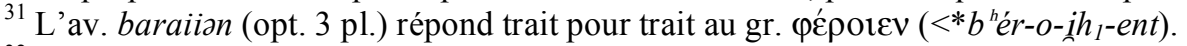

${ }^{32}$ Génitif d'attribution, consulter ReICHELT (1909 : 255). Noter ainsi le véd. déhi me « donne-moi ! ».

${ }^{33}$ Pour l'épithète $s \bar{u} r \bar{a}$ « la puissante » (= véd. *śúrā), consulter l'étude de LE FEUVRE $(2007: 124)$.

${ }^{34} \mathrm{~L}$ 'av. dātiia- veut dire « conforme au dāta-» (dâta- n. « loi » est ici à entendre au sens de « loi naturelle »).

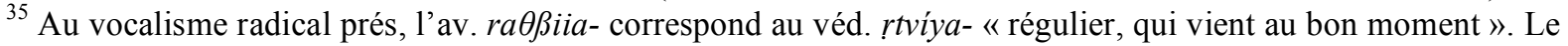
véd. rtú- m. peut désigner les époques de la femme (d'où le skr. cl. rtu-matī adj. f. « qui a ses règles »).

${ }^{36}$ Le hitt. $\bar{u} d a-$ «apporter » reflète i.-e. *aú- $d h_{3^{-}}$. KIMBALl $(1999: 225)$ pose un étymon i.-e. *ou qui ne tient pas compte du lat. $a u$-. Le degré zéro s'explique bien en partant du participe üdant- « apporté » (<*áúldh $\left.h_{3}-o n t-\right)$.

${ }^{37}$ Le mérite de cette explication revient à F. FLECK (ainsi que les exemples qui suivent). Qu'elle trouve ici l'expression de ma gratitude. Il est envisageable de dire que le lat. $d \bar{e}$ - recouvre en partie les emplois de $a u$-,

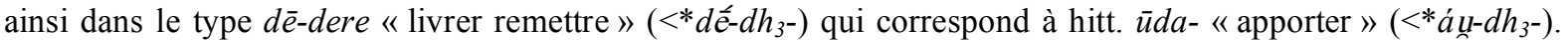
Avec un renouvellement formel de la racine (et non du préverbe), on peut considérer que le syntagme ancien conservé par le véd. áva-HAN- « terrasser, abattre » (<*áun-g $\left.g^{y h} e n-\right)$ serait indirectement reflété par le v.-sl. u-biti « tuer » (lequel présente la racine slave vivante pour « battre, frapper »).
} 
d'éloignement (ainsi en lat. dé-fugiō «s'enfuir»), de cassure (lat. dè-cìdere, fr. dé-capiter), ou de rapprochement (lat. dé-ferō «présenter, offrir ${ }^{38}$ ). L'évolution syntaxique précisant la nature du mouvement se surprend en plein développement dans les langues historiques : c'est ainsi que le véd. áva-TR̄- « descendre, s'incarner (dans un avatar) » finit par revêtir le sens de «détourner de ${ }^{39}$. Le tour se retrouve chez Kālidāsa, Rāgh., 6. 30ab,

\section{athāngarājād avatārya cakșur yāhīti janyām avadat kumārī}

« Mais la princessse, écartant ses yeux du roi des Angas : 'Viens !' dit-elle à sa compagne »

Il ne manque pas de trace du préverbe *áu indiquant un mouvement du haut vers le bas, ainsi dans le syntagme *áun-ped- «tomber, s'abattre » inférable de la correspondance entre le v.-sl. u-pad-o, u-pas-ti «tomber» et de l'av. auua.PAD- «tomber » attesté à l'infinitif en $Y$ 44. 4a,

\section{kasnā dərətā zq̨mčà adō nabåsčā auuapastōiš}

«Qui donc a fixé la terre en bas et les cieux (pour les empêcher) de tomber ${ }^{40}$ ?»

Fait notable, le préverbe áva-, nettement résiduel, est en distribution complémentaire avec l'usuel ní- «vers le bas » dans le participe privatif atharvavédique *an-ava-drā-ná« qui ne peut trouver le sommeil » en regard du groupe vivant de $n i-D R \bar{A}-$ « s'endormir» (avec son dérivé inverse $n i$ - $d r a ̂$ f. « assoupissement ») ${ }^{41}$. Il est est donc envisageable de poser trois valeurs pour i.-e. *áun : « de haut en bas », « au loin » et « auprès ».

\begin{tabular}{|c|c|c|}
\hline *áu « de haut en bas » & *áu « éloignement » & *áu « rapprochement » \\
\hline $\begin{array}{c}\text { *áu-ped-《 tomber» } \\
\text { v.-sl. u-pad-Q « tomber» } \\
\text { av. auua-PAD- « tomber » } \\
\text { véd. áva-PAD- « tomber » }\end{array}$ & 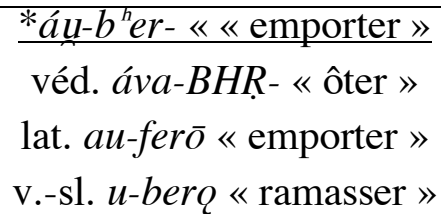 & $* a ́ u-b^{n} e r-\ll \ll$ apporter $»$ \\
\hline
\end{tabular}

\footnotetext{
${ }^{38}$ Dans le tour ad eum défertur imperium (Cæs., G., 7.4, 6) « on lui remet le commandement en chef ». Noter que $d \bar{e}$-ferō présente aussi le sens étymologique de "porter d'un lieu élevé dans un autre plus bas » (ainsi chez Liv., 22. 15, 12, castra in uiam dêferunt quā Hannibal ductürus erat « ils descendent leur camp en direction de la route par où hannibal devait passer $\gg)$.

${ }^{39}$ Avec un ablatif d'origine (ànga-rājād). L'absolutif ava-tār-ya présuppose un présent causatif *ava-tār-ay-a-ti « dé-tourner».

${ }^{40}$ L'infinitif au génitif d'origine se retrouve en védique, sur base de nom-racine, en $R V$ 2. 29, 6d, trádhvam kártād avapádo yajatrăh \# «sauvez (-nous) de la fosse, de tomber (dans elle), vous dignes du sacrifice! » (l'hymne s'adresse aux víśve devăh). Le tour fonctionne à la manière d'un double datif : il y a une attraction casuelle (il faut entendre « empêchez-nous de tomber dans la fosse! »).

${ }^{41}$ Attesté en $A V 8.1,13 \mathrm{~b}$, asvapnáśca tvānavadrānááśca rakșatām \# « que celui qui ne dort point ainsi que celui

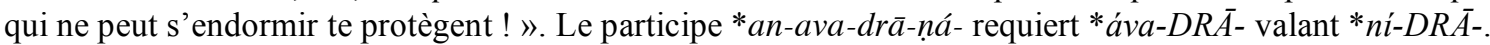




\begin{tabular}{|c|c|c|}
\hline 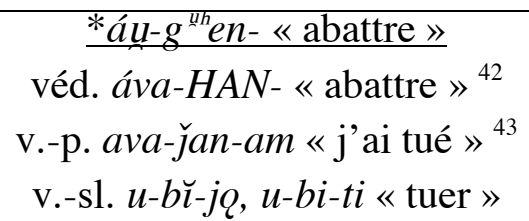 & 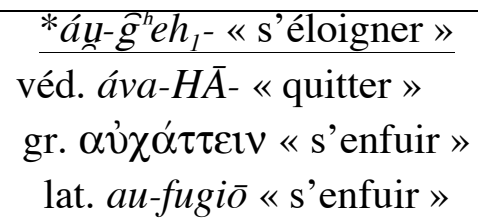 & *áú-deh $3^{-}$《 apporter » \\
\hline
\end{tabular}

5.5. une locution archaïque : véd. *áva divás « depuis le haut du ciel »

Si le lat. *auernus signifie « situé en bas » (par contraste avec supernus), il faut en rapprocher la préposition véd. áva « en bas, au loin » qui revêt parfois le sens de « depuis, du haut de », ainsi dans le tour archaïque *áva divás « depuis le haut du ciel » en $A V 7.55,1$ a (recension Śaunaka)

yé te pánthāno 'va divó yébhir víśvam áirayah

tébhị̣ sumnayâ dhehi no vaso

«Tes chemins qui partent du ciel, ceux par lesquels tu procures (*á-ìraya-s) toute chose, emprunte-les, ô Vasu (=Indra), pour nous placer dans ta faveur ! »

Il est à noter que les pada $^{44}$ ab se retrouvent dans le SV $1.172 \mathrm{ab}$, yé te pánthā adhó divó yébhir vyàsvam áirayah. Le terme vyàśvam, métriquement meilleur que vísvam ne fournit pas un sens cohérent. Il doit s'agir d'une erreur pour *vyàștam «le lot imparti à chacun » (sur vy-AŚ- « obtenir » et, pris intransitivement : «échoir en partage »). Le texte du $S V$ présente la préposition adhás « sous », ce qui doit représenter une modernisation du texte. Le textus traditus recouvre une couche plus archaïque, qui devait primitivement se présenter comme suit :

$$
\text { yé te *pánthā.o 'va divó yébhir *v(í)yașțam áirayah (8+8) }
$$

Il faut admettre qu'en devanāgarī, un nominatif pluriel archaïque de type *pánthā.as « les chemins » $\left(<\mathrm{i}\right.$.-e. * pént-oh -es $\left.^{45}\right)$ ne pouvait guère se noter autrement que *पन्थाओ. Il n'est pas interdit de penser que le copiste du $S V$ aurait altéré *ये ते पन्थाओ sव द्विवो (*yé te pánthā.o 'va divó) en ये ते पन्था अधो दिवो (yé te pántha adhó divó ) avec permutation du ो et remplacement de *รवे en 'ध pour rétablir un texte sanskrit correct. Le texte de l' $A V$ aurait donc tout simplement modernisé l'archaïque *pánthā.o en pánthāno (lesquels sont métriquement superposables).

\footnotetext{
${ }^{42}$ Noter la figura etymologica «jeter à terre » bien attestée par l'AV 13. 1, 30b, avācínān áva jahi « tue-les et mets-les à terre!». La forme athématique de l'adjectif ávāñc- «tourné vers le bas, situé en bas » est conservée dans le Jaim. Br. 1, 123, avāco 'vaghnan « ils les abattirent et leur firent joncher le sol». L'adjectif spatial ávāñc-indique donc le résultat (« être gisant»). L'amorce de ce tour est à chercher dans le $R V 4.25,6 \mathrm{~d}$, avahantéd ávācah \# (=*ava-HAN-tấ+íd) «il (scil. Indra) les jette à terre et les anéantit ».

${ }^{43}$ DB I : 57, avam gaumātam tyam magum avājanam « j’ai tué ce Gaumāta, le Mage ».

${ }^{44}$ Le pada est un terme de métrique indienne qu'on peut rendre par « hémistiche ». La désignation vient du fait que la strophe du sanskrit épique est un distique, dont les quatre hémistiches sont comme les quatre pattes (pada-) d'un animal. On les désigne commodèment par les lettres $a, b, c$ et $d$.

${ }^{45}$ Pour le paradigme i.-e. du nom du « chemin » se référer à WIDMER (2003: 139).
} 


\section{6. ávara- et av. aora « ici-bas »}

Reste à étudier la sphère d'emploi de l'adjectif contrastif i.-e. *áun-(e)ro- « situé vers le bas ». Il est manifeste que ni le véd. ávara- « situé en bas » ni l'av. aora « en bas, ici-bas » ne désignent à proprement parler des lieux infernaux. Ils désignent en revanche le monde d'ici-bas, qui est le monde inférieur pour les dieux. L'étude de la phraséologie ne laisse aucun doute là-dessus :

Yt 13. 147,

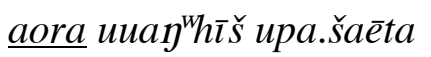
yå āpō yåsča $\bar{a}$ uruuarå yåsčā aș̆āunq̨m frauuaș̆aiiō

$R V$ 43. 34. 5c, kó addhâ veda ká ihá prá vocad dádṛ́ra eșām avamấ sádāmsi «Qui sait clairement, qui pourrait révéler ici bas quel chemin se porte en direction des dieux ?/

On voit d'eux (seulement) les séjours les plus bas »

\section{RV2. 9. 3b, ávare sadhásthe \#}

« dans ton séjour inférieur » $(=\text { sur terre })^{49}$

En revanche, la désignation du monde souterrain par un adjectif contrastif en *-eroest attestée en anatolien, avec les kattereš šiuneš «dieux infernaux » ${ }^{50}$. L'adjectif kattera« inférieur, infernal » (noté GAM-ra) est formé sur l'adverbe katta « en bas ». Le cas de figure rappelle les faits italiques, avec les dī infer $\bar{\imath}$ « dieux infernaux » (Cic.) qui s'opposent aux dī superi $\ll$ dieux du ciel ${ }^{51}$.

\section{6. bilan :}

Le nom de l'Averne s'explique bien en partant d'une formation d'adjectif contrastif en -ernus (<*-er-ino-) ${ }^{52}$, soit *auernus, - $a$, -um «situé en bas » apparenté au véd. ávara« inférieur ». L'it. com. *aú-er-o- « inférieur» (vs. *(s)uper-ino- « supérieur » $\left.{ }^{53}\right)$ serait à un adverbe *aun-er « au loin, loin de, en bas » ce que internus (<*enter-ino-) est à inter.

\footnotetext{
${ }^{46}$ Cognat du véd. vásvīh .

${ }^{47}$ Ce sont les anges gardiens de l'Avesta. Ils se confondent parfois avec l'âme des trépassés eux-mêmes.

${ }^{48}$ Cognat du véd. rtắ-van- « qui vit selon le rtá-, pieux, juste, vertueux ».

${ }^{49}$ Le destinataire de l'hymne est Agni, qui réside au ciel (AV6. 80. 3a, diví te sadhástham \#).

${ }^{50}$ Forme citée chez TISCHLER (2001: 76).

${ }^{51}$ Pour ces faits, consulter GARNIER (2008 : 132).

52 Pour les dérivés de ce type, voir l'étude de SZEMERÉNYI (1991).

${ }^{53}$ Lequel correspond étymologiquement à l'arm. verin « supérieur, situé en haut» (GARNIER, $2008: 148$, n. 52). Noter en outre la variante *uperi-tnó- reflétée par le skr. upari-tana- « supérieur ».
} 
Une preuve supplémentaire de la valeur spatiale du suffixe complexe *-er-inó- posé ici pour rendre compte du lat. Auernum serait le terme cauerna f. " cauité » évidemment en rapport avec l'adjectif cauus «creux ». L'emploi du suffixe fait songer à une collusion entre les deux familles, ce dont semble témoigner un passage de Lucrèce (6. 596- $8^{54}$ ),

Ancipit̄̄ trepidant igitur terrōre per urb̄̄s, tecta superne ${ }^{55}$ timent, metuunt inferne cauernās

terrā̄ nē dissoluat nātūra repente

«Une double terreur saisit alors les villes éperdues : craignant au-dessus d'elles la chute de leurs toits, elles redoutent encore que par-dessous la nature ne disjoigne et renverse soudain les cavernes souterraines. ${ }^{56}$

Il est donc envisageable de poser comme source du lat. cauerna « cavité souterraine » une sorte de $d v a n d v a$ syncopé *cauus $+*$ auernus « creux et profond $»{ }^{57}$. La plupart des mots en -erna du latin sont ambigus ou problématiques : lanterna $\mathrm{f}$. « lanterne » est une adaptation du gr. $\lambda \alpha \mu \pi \tau \eta \dot{\rho} \rho$ et taberna $\mathrm{f}$. «taverne » ne continue pas nécessairement un plus ancien *trab-erna «cabane en rondins ». Il n'est donc pas exclu de rendre compte du lat. cauerna par un ancien adjectif *cauernus, $-a$, - um « creux et profond ».

Romain GARNIER

Université de Limoges garromain@gmail.com

Éléments de bibliographie :

BRACHET J.-P. (1998), Les préverbes $A B-, D \bar{E}-, E X$ - du latin : étude linguistique, Paris 1998.

DÜNKEL G.E. (1988), « Laryngeals and Particles : *u $* h_{2} u$, and *awo», in Die Laryngaltheorie und die Rekonstruktion des indogermanischen Laut- und Formensystems. Ed. A. BAMMESBERGER, Heidelberg 1988, pp. 107-121.

GARNIER R. (2008) « Nouvelles réflexions étymologiques autour du gr. őv $\theta \rho \omega \pi \mathrm{s}$ », BSL 103/1, 2007 (2008), pp. 131-154.

\footnotetext{
${ }^{54}$ Il est question des tremblements de terre.

${ }^{55}$ La scansion exceptionnelle sŭpernĕ se retrouve chez Horace $(O ., 2.20,10)$. Il faut sans doute admettre ici le croisement des adverbes en $-\bar{e}$ de type hümāne issus d'anciennes finales d'instrumental $\left(<\mathrm{i} .-\mathrm{e} .{ }^{*}-e-h_{l}\right)$, correspondant en principe à des adjectifs de la première classe en $*_{-}-{ }_{-}$, avec les emplois du neutre de deuxième classe d'adjectifs, soit le type dulcĕ rīdens, qui est en voie d'adverbialisation. Il n'est pas impossible de partir du type běne qui repose sur un plus ancien *duenē en vertu de l'abrégement des mots ïambiques.

${ }_{56}^{5}$ Trad. A. ERNOUT, CUF, $1964^{2}$ (prem. éd. 1920).

${ }^{57}$ Il faut ici citer le fameux passage du cheval de Troie, chez Virg., En. 2, 19, penitusque cauernās \# ingentīs uterumque armātō mīlite complent «jusqu'en leurs profondeurs ils remplissent de soldats armés les cavités énormes, le ventre ». On retrouve ici associées les notions de « creux » et de « profondeur ».
} 
Kimball S. (1999), Hittite Historical Phonology, Innsbruck 1999.

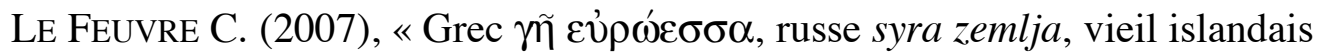
saurr, 'la terre humide' : phraséologie indo-européenne et étymologie », BSL 102/1, 2007, pp. 101-129.

LEUMANN M. (1977), Lateinische grammatik (LEUMANN - HOFMANN - SZANTYR), München 1977.

Melchert G. (1984), Studies in Hittite Historical Phonology, Göttingen 1984.

ReICHELT H. (1909), Awestisches Elementarbuch, Heidelberg 1909 (Dritte, unveränderte Auflage 1978).

RIX H. (1997), « Autumnus 'Herbst' und andere lateinische Vertreter der Wurzel *temh ${ }_{1}$ - 'schneiden' », in Scríbthair a ainm n'ogain : Scritti in Memoria di Enrico CAMPANILE, Ambrosini, RICCARDo et al. edd., Pisa, 1997, Vol. II, pp. $871-889$.

SOMMER F. (1914), Handbuch der lateinischen Laut- und Formenlehre, 2. und 3. Auflage, Heidelberg 1914.

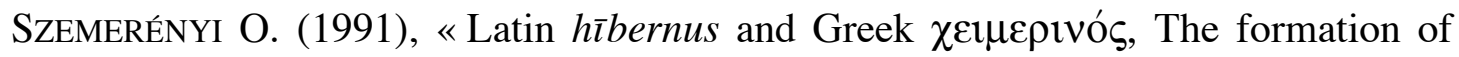
time adjectives in the Classical languages » (= Scripta Minora, Innsbruck 1991, Vol. II, pp. 1141-1159).

TISCHLER J. (2001), Hethitisches Handwörterbuch, Innsbruck 2001. de VAAN M.,

- (2003-04) «Latin au 'away', an allomorph of $a b »$, in Anuari de Filologia 25/26, Barcelona, 2003-04, pp. 1-7.

- (2008) Etymological Dictionary of Latin and the Other Italic Languages, Leiden 2008.

WACKERNAGEL-DEBRUNNER (1896-1954 I à IV), Altindische grammatik, (5 vol. : I Lautlehre, II,1, Wortlehre, II, 2, Die Nominalsuffixe, III, Nominalflexion, IV, Verbum und adverbium), Göttingen 1896 - 1954 (nouvelle édition de 1957, avec introduction générale par L. RENOU, 125 pp.). (abrév. AiGr.).

WidMER P. (2003), Das korn des weiten Feldes, Innsbruck 2003.

Sommaire: Dans cette contribution, on se propose d'étudier l'étymologie du nom de l'Averne. Il ne fait pas de doute que les anciens y voyaient la désignation d'un marais méphitique, réputé infranchissable aux oiseaux (d'où le recours à un étymon grec ôopvo૬). Le terme auernum semble un terme générique (noter Auerna loca chez Lucr. 6, 738) avant de désigner spécifiquement l'entrée des Enfers sise non loin de Cumes. Une nouvelle orientation étymologique consisterait à y voir un ancien adjectif spatial contrastif en -ernus (<*-er-inó-). Le terme serait apparenté au véd. ávara- «inférieur». On admettrait en ce cas un étymon it. com. *aun-er-ino- « inférieur ». Les loca auerna (sans majuscule) seraient des loca inferna.

\section{WHERE THE NAME OF THE LACVS AVERNVS COMES FROM ?} (facilis descensus Auerno)

Abstract: The following paper intends to make an etymological study of the lacus 
Auernus. No doubt is left that Roman authors themselves would explain its name by a farfetched folk-etymology, based upon a Greek compound őopvo $\zeta$ whicht is almost certainly a ghost-word. This would refer to the lake being regarded as a noxious swamp, causing the death of anything flying over. In fact, Latin auernum seems to have been a generic name (Auerna loca is met with in Lucretius 6, 738), before meaning the entrance of the underworld near Cumæ. A new etymological orientation might be to explain this word as a former adjective ending in -ernus (<PIE *-er-inó-), with the well-known oppositional suffix *-ero-. Latin *auernus would eventually match with Vedic ávara- "lower». Henceforth, one may assume a Com. Italic etymon *au-er-ino- «lower». The so-called loca auerna (without capital letter) would be nothing else but loca inferna. 